\title{
Abstracts presented at the Otology Section Meetings, Royal Society of Medicine, Entex Short Paper Prize, 3 February 2012 and 1 March 2013, London, UK
}

Abstracts for 2012

Outcomes of partial mastoid cavity obliteration

K McAllister

From the Monklands Hospital, Airdrie

\section{Introduction}

The management of extensive cholesteatoma often results in a canal wall down procedure with a mastoid cavity. Large cavities can lead to problems, including discharge and the need for repeated suction clearance. Mastoid cavity obliteration achieves a smaller cavity which can be self-cleansing and may achieve a high rate of dry ear.

Study aims

To examine whether the partial mastoid cavity obliteration technique achieves a smaller self-cleansing cavity than the standard mastoid cavity technique, and to assess patients' quality of life (QoL).

\section{Patients}

The study comprised consecutive patients who had undergone mastoid operations performed by a single surgeon over a two-year period (July 2009-May 2011).

\section{Methods}

Data from the international ear audit and operative records were used to identify all mastoid operations carried out during the study period. A total of 52 patients had undergone mastoidectomy during the study period. A partial mastoid cavity obliteration technique was used in 27 cases (51 per cent), and a standard mastoid cavity technique was employed in 25 cases. In 24 out of 27 patients with mastoid cavity obliteration, only conchal cartilage was used. In three cases, Osteoset ${ }^{\circledR}$ pellets or Kasios ${ }^{\circledR}$ granules were used in addition to conchal cartilage. Patients were regularly followed up in the out-patient department and post-operative clinical findings were documented. Assessments of QoL were made using the Glasgow Benefit Inventory, which is a well-validated tool.

\section{Results}

A dry ear was achieved in 93 per cent of cases (an equivalent dry ear rate for the two techniques). There was a 75 per cent response rate for the Glasgow Benefit Inventory. This revealed a better QoL for patients that underwent partial mastoid cavity obliteration compared with those who underwent the standard mastoid cavity technique (Glasgow Benefit Inventory total score 24 vs 19, general subscale score 27 vs 15).
Conclusions

Partial mastoid cavity obliteration is a quick and simple technique that appears to achieve a much smaller cavity than the standard mastoid cavity technique and an improvement in QoL.

Evidence for early loading of the bone-anchored hearing aid BI300 system (at four weeks post-surgery)

C McLarnon

From the Freeman Hospital, Newcastle upon Tyne

\section{Introduction}

This study aimed to directly measure the stability of the bone-anchored hearing aid (BAHA) abutment using resonance frequency analysis and to report on the change in stability over time for implants loaded with the sound device four weeks post-operatively.

\section{Methods and materials}

The principle behind resonance frequency analysis is to obtain a numerical value relating to stability. A Smartpeg (a $1 \mathrm{~cm}$ commercially manufactured attachment) is screwed onto the abutment, and its resonance in a magnetic field is measured with an Osstell recording device. The degree of movement (vibration) is inversely proportional to the stability of the abutment; the implant stability quotient is the numerical figure derived from this. Resonance frequency analysis measurements were obtained at the time of implant surgery, and at 1 week, 4 weeks and 16 weeks post-surgery. Patients were fitted with the new Cochlear ${ }^{\mathrm{TM}}$ BAHA $^{\circledR}$ BI300 series implant using a one-stage procedure; this system was loaded four weeks post-operatively.

\section{Results}

A total of 71 consecutive patients (with a male to female ratio of 25:46) were prospectively recruited. Twenty patients had bilateral BAHAs fitted, giving a total of 91 implants. Average implant stability quotient change (delta) for the cohort gave a value of 0 at the time of surgery. At 1 week, this value was -0.07 (95 per cent, confidence interval $(\mathrm{CI})=0.73$ ), at 4 weeks it was 1.22 (95 per cent, $\mathrm{CI}=$ $0.58)$ and at 16 weeks it was $1.6(95$ per cent, $\mathrm{CI}=0.77)$.

\section{Discussion}

The resonance frequency analysis results suggested immediate stability of the BI300 abutment (at the time of surgery); furthermore, this stability was maintained. The data supported the evidence for early loading (at four weeks postsurgery), indicating good clinical safety. 
Assessment of service quality in an otology clinic using a modified 'Servqual' questionnaire

J Wasson

From the Norfolk and Norwich University Hospital

Objectives

Gap 5 of the gap model of service quality proposes that service quality can be evaluated based on the size and direction (positive $v s$ negative) of disconfirmation (gap) between a consumer's expectations and their perceptions of the service received. The modified 'Servqual' questionnaire is a validated tool used to measure gap 5 in healthcare services. This questionnaire was used to assess service delivery in the otology clinic, both from a patient and frontline staff perspective.

\section{Methods}

The Servqual questionnaire is a 22-item tool covering 5 dimensions of service quality (tangibles, reliability, responsiveness, assurance and empathy). It comprises two parts, which assess service expectations and perceptions of the otology clinic service. The questionnaire was distributed to 40 patients and 12 frontline staff in an otology clinic. This enabled us to compare and contrast consumer and provider gap scores (perception score minus expectation score) in service quality.

\section{Results}

Out of the 22 scores attained, 19 of the patient gap scores and 20 of the staff gap scores were negative, indicating less than satisfactory service delivery, from both a patient and staff perspective. Staff gap scores were more negative than patient gap scores, indicating staff pessimism regarding process failure and service strain. Gap scores for empathy were positive for staff, but negative for patients. This is concerning as it suggests that staff believe the service quality provided is acceptable in terms of empathy when in fact patients perceive this aspect of service quality to be unacceptable.

\section{Conclusions}

The Servqual questionnaire is a validated tool for identifying perception-expectation gaps in the quality of the otology clinic service. The size and direction of these gaps can help to prioritise incremental change in order to improve the quality of the service delivered.

\section{Abstracts for 2013}

Finding the most effective cerumenolytic agent

C Saxby, R Williams, S Hickey

From the Torbay Hospital, Torquay

Aim

To conduct an in vitro study to determine the most effective over-the-counter topical cerumenolytic agent.

\section{Methods}

Cerumen was collected from patients who attended the ENT out-patient clinic. The cerumen was mixed and made into a homogeneous ball. Discs of wax were punched out using the end of an otoscope speculum; these were weighed to produce samples that were uniform in shape and size. Each cerumen sample was placed into a tube which contained
$5 \mathrm{ml}$ of one of six cerumenolytic preparations. Two oilbased and four aqueous-based agents were tested.

The tubes were observed at 30 minutes, 3 hours and 12 hours. Digital photographs were taken to record the amount of cerumen disintegration. The cerumen discs were removed from the solutions at 12 hours, dried at room temperature for 48 hours and then re-weighed. The experiment was then repeated two times.

\section{Results}

Distilled water was the best agent for reducing cerumen disc mass. The distilled water and sodium bicarbonate tubes showed substantial disintegration at 12 hours. The tubes with the oil-based agents did not show any visible disintegration, and there was no decrease in the dried weight of the cerumen discs.

\section{Conclusion}

Distilled water caused the greatest degree of cerumenolysis. Oil-based cerumenolytic agents were ineffective.

Whole exome sequencing identifies novel genetic variants in familial otosclerosis

J L Ziff, J Lavy, H Powell, S Khalil, K P Steel, S R Saeed, S J Dawson

From the University College London Ear Institute

Otosclerosis is a common acquired form of conductive hearing loss, characterised by abnormal bone remodelling within the otic capsule, leading to fixation of the stapes. The aetiology of otosclerosis is unknown, but both environmental and genetic factors have been implicated in disease pathogenesis. A number of investigative strategies have been utilised to identify genes involved in the disease process; however, no definitive causative genes have been identified.

Whole exome sequencing was performed (using next generation sequencing technology) on 10 individuals across 4 different families, each of which exhibited monogenic inheritance of otosclerosis. The study aimed to identify the pathogenic variant in each of the four families. The individuals examined were: two non-immediate family members with otosclerosis from each of two unrelated Caucasian British families; three immediate family members with otosclerosis from a third family with multiple consanguineous marriages; and two affected and one unaffected sibling from a fourth family. On average, 90000 variants were mapped per individual.

Variants presenting exclusively in the affected members of each family were identified. A filtering strategy was designed to remove known common variants and prioritise those most likely to be involved in disease pathogenesis. Prioritisation was given to rare variants at conserved sites that were predicted to have a damaging effect on the resulting protein. Based on this analysis, approximately five candidate variants were selected in each family for Sanger sequencing in additional family members, to confirm those variants associated with the disease.

Genotyping assays will be conducted in an otosclerosis cohort recruited at the Royal National Throat, Nose and Ear Hospital in London. The presence of any of the variants in additional cases of familial otosclerosis will provide strong evidence for a role of these genes in the disease pathology of otosclerosis. 
The best seat for the hearing impaired within a noisy restaurant

\section{S J C Fishpool, S S Backhouse}

From the Princess of Wales Hospital, Bridgend

\section{Introduction}

There are more than 10 million people with some form of hearing loss in the UK. Social isolation is a significant aspect of this disability. We wanted to address an 'everyday' situation of going to a restaurant with a partner. What would be the 'best seat in the house' for a hearing-impaired individual to hear their partner?

\section{Method}

A simple plan of a restaurant was presented to four professional groups (ENT surgeons at the British Society of Otology Annual Meeting 2012, medical students studying at our hospital, general practitioners at a regional training programme meeting and audiologists at a national audiology conference). Each was asked to mark on the plan the 'best seat in the house' for both a symmetrically and asymmetrically hearing-impaired individual. The results were compared to the 'gold standard' of a theoretical audiological model.

Results

The correct seat was predicted by 35 per cent of medical students $(n=16), 34$ per cent of audiologists $(n=45), 34$ per cent of ENT surgeons $(n=36)$ and 13 per cent of general practitioners $(n=15)$. The only statistical difference was between the general practitioners and the other groups.

\section{Discussion}

The better performance of ENT surgeons and audiologists over general practitioners in this study was as expected. The medical students performed particularly well, however, and this may be reflective of the greater care they took in answering the questions. We hope that continued improvements in our understanding of how speech in noise is perceived will aid in the counselling, selection and clinical outcomes of patients.

Systematic review of cochlear implantation in children with developmental disability

N Eze, E Ofo, A Fitzgerald O'Connor, D Jiang

From the St Thomas' Hospital, London

\section{Objective}

To perform a systematic review, comparing the outcomes of cochlear implantation in children with or without developmental disability.

\section{Data sources}

Medline, Embase and Cochrane databases were searched for relevant articles published from 1950 (or the start date of each database) onward. The search was performed on 1 November 2012, and included articles published ahead of print. There were no language restrictions.

\section{Study selection}

The initial search produced 441 articles, of which 13 met the inclusion criteria. The articles focused on children with cochlear implants and developmental disability, whose expressive and/or receptive language outcomes were compared to those of children with cochlear implants and normal development.

\section{Data extraction}

Study quality (for each article) was assessed on the basis of whether: ethical approval was gained, the study was prospective, eligibility criteria were specified, appropriate controls were used, adequate follow up was achieved and outcome measures were defined. Cochlear implant outcome measures included expressive and receptive speech and language development, quality of life, and behaviour.

\section{Data synthesis}

Because of the heterogeneity of the post-operative follow-up periods and outcome measures reported, it was not possible to pool the data and perform a meta-analysis. Therefore, comparisons were made via a structured review.

\section{Conclusion}

Seven out of the 13 studies assessed demonstrated a worse outcome for children with developmental disability. The remaining six articles showed no differences in the postimplantation outcomes between the two groups. There may be no apparent benefits of cochlear implantation in children with developmental disability when traditional assessment tools are used to evaluate outcomes; however, cochlear implantation appears to improve their environmental awareness and quality of life. Further work is needed to define the term 'benefit' when used in this context for this vulnerable group. Implantation appears to have a negative impact on children with autistic spectrum disorder.

Limited eosinophilic granulomatosis with polyangiitis, with primary otological manifestations - a new disease subset

J Der Kureghian, N Donnelly, P Axon, P Jani, D Jayne

From the Royal Free Hospital, London, University College London, and Addenbrooke's Hospital, Cambridge

\section{Introduction}

Eosinophilic granulomatosis with polyangiitis is a vasculitic disease characterised by asthma, eosinophilia and extravascular eosinophilic granulomas. Otological involvement has been rarely described in patients with this condition.

\section{Objective}

To describe a limited form of eosinophilic granulomatosis with polyangiitis in which otological manifestations predominate.

\section{Study design}

A prospective analysis of 60 patients with suspected or confirmed eosinophilic granulomatosis with polyangiitis, with otolaryngological involvement, was carried out. All cases with predominantly otological manifestations were analysed.

Results

Seven patients were identified. All patients were female, with a median age of 50 years. In all cases, otological involvement occurred in the early stages of the disease and led to the patients' diagnosis. Patients presented with: conductive hearing loss; tympanic perforations; thick, tenacious otorrhoea; and middle-ear granulation tissue. All patients responded to glucocorticoid treatment.

\section{Conclusions}

We propose that the series of patients described represent a novel disease subset. There is likely to be under-diagnosis 
of this condition. Greater awareness of limited eosinophilic granulomatosis with polyangiitis would permit earlier diagnosis and treatment, better hearing outcome, and the prevention of permanent sequelae.

Delineating the role of integrins in the repair and

K Hussain, S Saeed, S Dawson, A Forge, R Taylor

From the University College London Ear Institute

The vestibular system, housed in the otic capsule of the petrous temporal bone, is made up of five end organs that subserve balance: the three semi-circular canals, the utricle and the saccule. The sensory neuroepithelia of these tissues comprise mechanoreceptive, sensory hair cells and supporting cells.

The loss of hair cells in the vestibular system results in severe balance deficits. Dizziness is the most common cause of visits to the general practitioner amongst patients aged over 75 years. Furthermore, vestibular dysfunction has been shown to be a significant contributor to falls in the elderly, which have been estimated to cost the UK health and social services budgets almost $£ 1$ billion per year. Understanding how this tissue responds to injury will have a direct impact for developing future therapies.

Integrins are transmembrane receptors responsible for physiological and pathological processes throughout the body. Integrins comprise 18 alpha and 8 beta subunits, and form 24 obligate alpha and beta heterodimeric members. The latter mediate the interaction of cell-cell signalling, and communication between the cell and extracellular matrix.

Following the death of a vestibular hair cell, the lesion created is closed by the supporting cells via a process that requires cell shape changes and spreading. Such a process is likely to involve integrins, as is reported for wound healing in skin. A phagocytic process then follows in order to remove these dead hair cells. Certain integrin receptors recognise apoptotic cells and mediate phagocytosis. A limited number of hair cells are spontaneously regenerated, possibly due to the direct phenotypic conversion of the supporting cell into hair cells without an intervening mitotic event. We hypothesise that integrins play a role in repair and recovery processes in the vestibular sensory epithelia. We are currently testing this hypothesis with human vestibular tissue obtained from patients undergoing translabyrinthine procedures for acoustic neuromas.

Initial work has focused on identifying those integrins that are normally expressed in human vestibular tissues.

Quantitative polymerase chain reaction was used to screen for the integrin messenger (m)RNAs specifically expressed in human vestibular explants. After harvesting the tissue from the patient, the tissue was immediately transferred to RNAlater $^{R}$ solution for transport to the laboratory. The RNA was subsequently prepared using an RNeasy kit (Qiagen, Valencia, California, USA) and reverse transcribed into complementary DNA. Each array was performed with complementary DNA from the vestibular tissue explanted from a single patient. Data were collated from individual arrays and relative expression was quantified by quantitative polymerase chain reaction using the $\Delta \Delta$ threshold cycle method. The expression of specific integrin proteins was then localised (based upon the results of this analysis) using immunohistochemistry.

The results demonstrated that integrin subunit mRNAs ITA5, ITA6, ITA8, ITAV, ITB1 and ITB5 were expressed above background levels, suggesting their presence in human vestibular tissue. Previous work in our laboratory has identified these integrins in adult mouse vestibular tissue. Immunohistochemistry has demonstrated their localisation in human tissue in patterns comparable to those in mice.

This novel project identifies the integrins expressed in human vestibular tissue. The integrin profiles of human and mouse vestibular systems are similar but not identical. Work will continue to examine integrin protein expression and location, and to further elucidate their role in the repair of inner-ear sensory epithelia and hair cell regeneration by examining their relative expression at specific time points during culturing experiments. 